\title{
Correction to: Criticism of Triple Bottom Line: TBL (With Special Reference to Sustainability)
}

\author{
Amit Kumar Srivastava $^{1}$ (1) $\cdot$ Shailja Dixit ${ }^{2} \cdot$ Akansha Abhi Srivastava $^{3}$
}

Published online: 30 April 2021

(c) Springer Nature Limited 2021

\section{Correction to: Corporate Reputation Review https://doi.org/10.1057/s41299-021-00111-x}

Due to an unfortunate oversight the affiliation of Amit Kumar Srivastava has been given erroneously. It should read:

Amit Kumar Srivastava, Research Scholar, Amity Business School, Amity University Uttar Pradesh, Lucknow, India.

The original article has been corrected.

Publisher's Note Springer Nature remains neutral with regard to jurisdictional claims in published maps and institutional affiliations.

The original article can be found online at https://doi.org/10.1057/ s41299-021-00111-x.

Mr. Amit Kumar Srivastava amitmphil06@yahoo.com

DR. Shailja Dixit

shailjadixit1@gmail.com

Ms. Akansha Abhi Srivastava

srivastava.akansha09@gmail.com

1 Research Scholar, Amity Business School, Amity University Uttar Pradesh, Lucknow, UP, India

2 Associate Professor, Amity Business School, Amity University Uttar Pradesh, Lucknow, India

3 Assistant Professor, Institute of Hotel Management (IHM), Lucknow, UP, India 